
\title{
3 Research Square \\ The Protective Effect of Carvacrol on Acetaminophen-Induced Renal Damage in Male Rats
}

\section{Alireza Najafizadeh}

Rafsanjan University of Medical Sciences

Ayat Kaeidi

Rafsanjan University of Medical Sciences

Mohammadreza Rahmani

Rafsanjan University of Medical Sciences

\section{Elham Hakimizadeh}

Rafsanjan University of Medical Sciences

jalal Hassanshahi ( $\square$ hasanshahij@gmail.com )

Rafsanjan University of Medical Sciences https://orcid.org/0000-0003-3754-8152

\section{Research Article}

Keywords: acetaminophen, oxidative stress, apoptosis, carvacrol, antioxidant, protective effect

Posted Date: September 7th, 2021

DOl: https://doi.org/10.21203/rs.3.rs-758982/v1

License: (c) (1) This work is licensed under a Creative Commons Attribution 4.0 International License. Read Full License

Version of Record: A version of this preprint was published at Molecular Biology Reports on January 12th, 2022. See the published version at https://doi.org/10.1007/s11033-021-06985-8. 


\section{Abstract}

Acetaminophen overdose causes renal injury via oxidative stress and apoptosis induction. Carvacrol has antioxidant effect. The aim of this study was to determine the protective effect of carvacrol on acetaminophen-induced renal damage in male rats. In this experimental study, forty male Wistar rats were randomly divided to five groups $(\mathrm{n}=8)$ including control, carvacrol $10 \mathrm{mg} / \mathrm{kg}$, acetaminophen, acetaminophen + carvacrol $5 \mathrm{mg} / \mathrm{kg}$, and acetaminophen + carvacrol $10 \mathrm{mg} / \mathrm{kg}$. Animals initially received a single dose of acetaminophen $(500 \mathrm{mg} / \mathrm{kg}$ ), then were treated with carvacrol for one week (daily). Afterwards, renal blood flow (RBF), mean arterial pressure (MAP), renal perfusion pressure (RPP), renal vascular resistance (RVR), blood urea nitrogen (BUN), and serum creatinine were measured. Also, malondialdehyde (MDA) concentration, glutathione peroxidase (GPx) and superoxide dismutase (SOD) activity levels were measured in the kidney tissue. Hematoxylin and eosin method was used for histological assessment. The western blotting analysis was used to determine the Bax, Bcl-2 and cleaved caspase-3 proteins expression level in the kidney tissue. Carvacrol $(10 \mathrm{mg} / \mathrm{kg})$ could significantly increase the RBF, GPx and SOD activities and also reduced the RVR, serum creatinine, BUN, and MDA in the acetaminophen + carvacrol $10 \mathrm{mg} / \mathrm{kg}$ group versus acetaminophen group $(P<0.05)$. Also, carvacrol significantly decreased the cleaved caspase-3, Bax proteins expression level, and also kidney tissue damage score in the acetaminophen + carvacrol $10 \mathrm{mg} / \mathrm{kg}$ group versus acetaminophen group $(P<0.05)$. This study showed that carvacrol can attenuate the acetaminophen induced acute kidney damage via suppressing oxidative stress, apoptosis and its antioxidant effects.

\section{Introduction}

Currently, acetaminophen (paracetamol) is widely applied as a febrifuge and analgesic drug worldwide, but it induces kidney injury at high doses administration [1]. So that, acetaminophen overdose causes renal tubular damage and uremia in animals and humans [2] and it induces renal injury at least in 1-2\% of patients [3]. It has been shown that acetaminophen analgesic metabolites (Paracetamol is a phenacetin metabolite) have a nephrotoxic effect at high dose administration [2]. Acetaminophen overdose is a current type of toxic ingestion throughout the worldwide [3]. Although the mechanisms involved in renal injury following high-dose acetaminophen administration are not well understood, it has been shown that $\mathrm{N}$-acetylcysteine is used to treat acetaminophen-induced hepatotoxicity. $\mathrm{N}$ acetylcysteine increases the hepatic glutathione stores but is not able to protect the kidneys against acetaminophen overdose administration [4]. The molecular mechanism of nephrotoxicity is probably different from hepatotoxicity [2]. It has been shown that, taking high doses of acetaminophen weakens the kidneys' antioxidant system and causes acute kidney damage [5]. Moreover, it has been reported that acetaminophen induces apoptosis by activating the caspase cascade [2]. Today there is a great tendency to use herbal medicines to alleviate the side effects of various diseases because in addition to their protective effects, they reduce the risk of side effects caused by the use of chemical drugs [6-8]. Along with classic antioxidants in plants, phenolic compounds have been identified as important antioxidants [9]. Carvacrol is a main component of essential oils of phenolic compounds. Carvacrol is a monoterpenic 
phenol and exists in various plants such as oregano and thyme [10]. Carvacrol has various biological properties such as antioxidant, antibacterial, antifungal, anti-cancer, anti-inflammatory, antispasmodic, and vasodilator [11]. In addition, it has been shown that by increasing free radicals in the body, an imbalance is created between oxidants and antioxidants, which is defined as oxidative stress, leading to oxidative damage [12]. In this regard, it has been observed that carvacrol can effectively neutralize free radicals such as peroxyl radicals, superoxide radicals, and hydrogen peroxide [13]. Carvacrol has renoprotective effect via its phenolic hydroxyl group in renal ischemia-reperfusion model [14]. Since acetaminophen overdose administration can induce acute renal damage, accordingly, this study was designed to evaluate the protective effect of carvacrol on acetaminophen-induced renal damage in male rats.

\section{Material And Methods}

\section{Animals}

In this experimental research, forty male Wistar rats (210 $\pm 5 \mathrm{~g}$, eight weeks) were purchased from Rafsanjan University of Medical Sciences Animal House, Rafsanjan, Iran. The animals were maintained in a standard room (temperature: $23 \pm 1^{\circ} \mathrm{C}$, humidity: $50 \%$ ) with $12 \mathrm{~h}$ light/dark cycle. The rats were allowed free access to a standard rodent chow diet and drinking water. This study was approved by Ethics Committee of Rafsanjan University of Medical Sciences (Ethics No. IR.RUMS.REC.1397.222). Also, this research was carried out according to the animals care and use guidelines (National Institutes of Health Publication No. 85 - 23) revised in 2010.

\section{Experimental groups and protocol}

After the adaptation period (one week), rats were randomly assigned to five groups ( $n=8$ per group) including:

Group 1: Control.

Group 2: (Carvacrol): Animals in this group received carvacrol $(10 \mathrm{mg} / \mathrm{kg})$ for one week (daily) via intraperitoneal (i.p.) injection [15].

Group 3: (Acetaminophen): this group received acetaminophen $(500 \mathrm{mg} / \mathrm{kg})$ in a single dose by i.p. injection [3].

Group 4: (Acetaminophen + carvacrol $5 \mathrm{mg} / \mathrm{kg}$ ): this group initially received a single dose of acetaminophen (500 mg/kg, i.p.). Then rats were treated with carvacrol (5 mg/kg, i.p.) one hour later for one week (daily).

Group 5: (Acetaminophen + carvacrol $10 \mathrm{mg} / \mathrm{kg}$ ): this group initially received a single dose of acetaminophen $(500 \mathrm{mg} / \mathrm{kg}$, i.p.). Then rats were treated with carvacrol $(10 \mathrm{mg} / \mathrm{kg}$, i.p.) one hour later for one week (daily) [15]. 
A single dose of acetaminophen ( $500 \mathrm{mg} / \mathrm{kg}$, i.p) was used in this study [3]. In the treatment groups, rats first received high-dose of acetaminophen and then were treated with carvacrol ( 5 or $10 \mathrm{mg} / \mathrm{kg}$, i.p) for one week [15]. The animals in the control group received no intervention. The animals in group 2 received only carvacrol $(10 \mathrm{mg} / \mathrm{kg})$ without acetaminophen. Finally, 24 hours after the last intervention, the rats were weighed and anesthetized with urethane (1.7 g/ kg, i.p.) (Sigma, St. Louis USA) to measure the hemodynamic parameters.

\section{Hemodynamic parameters measurement}

At first, the rats' trachea was intubated via polyethylene tube (Microtube Extrusions, Australia) to facilitate breathing [16]. Then, two polyethylene tubes were used for catheterizing the left carotid and femoral arteries [17]. Mean arterial pressure (MAP) and renal perfusion pressure (RPP) were assessed via these catheters jointed to two transducers linked to PowerLab hardware (ADInstruments, Australia) and lab chart software. Also, the left renal artery was exposed and RBF (as perfusion units (PU)) was measured by help of a laser-Doppler perfusion monitor instrument (DRT4, Moor Instruments, UK) [18]. The hemodynamic parameters were recorded for thirty minutes, then the last five minutes of recording time were used for analysis [19]. RPP to RBF ratio was applied for the calculation of the RVR (mm $\mathrm{Hg}$ /perfusion units) indicator [18]. During the measurement period, the rat's body temperature was sustained at $37^{\circ} \mathrm{C}$ via a heated platform.

\section{Blood urea nitrogen and creatinine measurement}

After hemodynamic parameters measurement, heart puncture was used for blood samples collection. Then rat's blood samples were centrifuged at $6000 \mathrm{rpm}$ for $20 \mathrm{~min}$. The blood urea nitrogen (BUN) and creatinine $(\mathrm{Cr})$ concentrations were evaluated via quantitative determination kits (Pars Azmoon, Iran).

\section{Oxidative stress parameters assessments}

The rats were killed in deep anesthesia. Kidneys were removed. One of them was homogenized in ice-cold buffer solution and centrifuged ( $20 \mathrm{~min}$ at $6000 \mathrm{rpm}$ ). The supernatant was prepared for oxidative and apoptosis parameters. Some of the oxidative stress parameters like malondialdehyde (MDA) concentration, glutathione peroxidase (GPX) and superoxide dismutase (SOD) activity levels were measured in the kidneys using their commercial kits (Zellbio-Germany) [20-22].

\section{Histopathological assessments}

The other kidney was kept in formalin (10\%) for histological assessment. Then histological staining was done via hematoxylin and eosin (H\&E) method. Kidney tissue damage score (KTDS) was applied for histopathological assessments. KTDS was considered based on the tubular vacuolization and dilatation, debris, hyaline cast, interstitial edema and interstitial infiltration in the kidney tissue [23, 24]. The slides were graded from zero to three based on intensity of kidney tissue damage for each sample (0-0.5 = normal, $1=$ minor damage, $2=$ moderate damage, $3=$ severe damage) $[6,7]$.

\section{Apoptosis parameters measurement}


The immunoblotting method was done to measure the Bax, Bcl-2 and cleaved caspase 3 proteins expression level in the kidney tissue. In brief, each protein sample was separated from $12.5 \%$ polyacrylamide gel electrophoresis and transferred to polyvinylidene difluoride membrane. Each membrane was incubated overnight (at temperature of $4^{\circ} \mathrm{C}$ and $\mathrm{pH} 7.4$ ) in Tris-buffered saline and Tween-20 (20 mM Tris- $\mathrm{HCl}, 150 \mathrm{mM} \mathrm{NaCl}, 0.1 \%$ Tween 20$)$ with $5 \%$ nonfat milk. Then polyvinylidene difluoride membranes were incubated with rabbit polyclonal anti-Bax (1:1000), monoclonal rabbit anticaspase-3 (1:1000), and rabbit polyclonal anti-Bcl-2 (1:1000) antibodies for $3 \mathrm{~h}$ at temperature of 20 to $22^{\circ} \mathrm{C}$. Subsequently, each blot was washed with $20 \mathrm{mM}$ Tris- $\mathrm{HCl}, 150 \mathrm{mM} \mathrm{NaCl}, 0.1 \%$ Tween 20 (three times), and incubated with horseradish peroxidase-conjugated anti-rabbit secondary antibody (Abcam, 1:5000) at room temperature for another hour. Subsequently, blots were detected via an enhanced chemiluminescence method. Band densitometry analysis was done by the ImageJ software. Beta ( $\beta$ )actin (1:5000) was considered as a loading control.

\section{Statistical Analysis}

All data were statistically analyzed by GraphPad Prism version 6.01 for Windows (GraphPad Software, USA). Results are presented as mean \pm SD. For comparison between the groups (in quantitative values) One-way ANOVA followed by post hoc Tukey test was used. Moreover, the Kruskal-Wallis test was used for KTDS data analysis between the groups. The null hypothesis was rejected at the level of 0.05 .

\section{Results}

\section{The effect of carvacrol on body weight, renal hemodynamic and functional parameters}

Based on our results, no significant difference was seen between animal body weights at the end of the study. A single dose of acetaminophen $(500 \mathrm{mg} / \mathrm{kg})$ significantly decreased the RBF in the acetaminophen administered rats compared to the control group $(P<0.05$, Table 1$)$. Furthermore, carvacrol $(10 \mathrm{mg} / \mathrm{kg})$ could significantly increase the RBF in the acetaminophen + carvacrol $10 \mathrm{mg} / \mathrm{kg}$ group compared to acetaminophen administered rats $(P<0.05$, Table 1$)$. Also, taking high doses of acetaminophen $(500 \mathrm{mg} / \mathrm{kg})$ increased the RVR in the acetaminophen administered rats compared to the control group; however, this change was not statistically significant (Table 1). Moreover, carvacrol (10 $\mathrm{mg} / \mathrm{kg}$ ) could significantly reduce the RVR in the acetaminophen + carvacrol $10 \mathrm{mg} / \mathrm{kg}$ group compared to acetaminophen administered rats $(P<0.05$, Table 1$)$. However, indicators such as MAP and RPP did not change significantly in different groups (Table 1).

Our result also showed that serum creatinine and BUN increased in acetaminophen $(500 \mathrm{mg} / \mathrm{kg})$ administered animals when compared to control $(P<0.001$ and $P<0.01$ respectively, Table 1$)$. Furthermore, carvacrol $(10 \mathrm{mg} / \mathrm{kg})$ significantly decreased the serum creatinine and BUN in the acetaminophen + carvacrol $10 \mathrm{mg} / \mathrm{kg}$ group compared to acetaminophen administered rats $(P<0.001$ and $\mathrm{P}<0.05$ respectively, Table 1 ). Also, carvacrol at the dose of $5 \mathrm{mg} / \mathrm{kg}$ could decrease the serum 
creatinine level in the acetaminophen + carvacrol $5 \mathrm{mg} / \mathrm{kg}$ group versus acetaminophen administered rats $(P<0.05$, Table 1$)$.

\section{The effect of carvacrol on MDA concentration, GPx and SOD activities}

Free radical injury (using lipid peroxidation) was evaluated after administration of acetaminophen (500 $\mathrm{mg} / \mathrm{kg}$ ), which was considered as MDA levels. According to Fig. 1A, a single dose of acetaminophen significantly increased the kidney tissue MDA concentration in acetaminophen $(500 \mathrm{mg} / \mathrm{kg})$ administered rats rather than the control group $(P<0.05)$. Treatment of the rats with carvacrol $(10 \mathrm{mg} / \mathrm{kg})$ could decrease the kidney tissue MDA concentration in the acetaminophen + carvacrol $10 \mathrm{mg} / \mathrm{kg}$ group compared to acetaminophen administered group $(P<0.05$, Fig. $1 A)$.

In addition, acetaminophen $(500 \mathrm{mg} / \mathrm{kg}$ ) significantly decreased the GPx and SOD (two antioxidant enzymes) activity levels in the kidneys tissue of acetaminophen administered rats compared to the control group $(p<0.01$ and $p<0.001$ respectively, Fig. 1B and 1C). Treatment of the rats with carvacrol $(10 \mathrm{mg} / \mathrm{kg})$ increased the GPx and SOD activity levels in the kidneys tissue in the acetaminophen + carvacrol $10 \mathrm{mg} / \mathrm{kg}$ group versus acetaminophen administered group $(\mathrm{p}<0.05$, Fig. 1B and $1 \mathrm{C})$. Also, carvacrol at the dose of $5 \mathrm{mg} / \mathrm{kg}$ could increase the SOD activity level in the kidney tissue of the acetaminophen + carvacrol $5 \mathrm{mg} / \mathrm{kg}$ rats compared to acetaminophen administered group $(p<0.05$, Fig. 1C).

\section{The effect of carvacrol on renal apoptosis}

The results also showed that acetaminophen $(500 \mathrm{mg} / \mathrm{kg}$ ) markedly increases the cleaved caspase-3 ( $P$ $<0.01)$, Bax $(P<0.001)$ proteins expression level, and also decreases the $\mathrm{Bcl}-2$ protein expression level $(\mathrm{P}$ $<0.05$ ) in the kidneys tissue of acetaminophen administered rats compared to the control (Fig. 2). Moreover, carvacrol $(10 \mathrm{mg} / \mathrm{kg}$ ) could prevent acetaminophen-induced cleaved caspase-3 and Bax proteins expression rate in the kidney tissue of acetaminophen + carvacrol $10 \mathrm{mg} / \mathrm{kg}$ group compared to acetaminophen administered group ( $p<0.05$, Fig. 2A and 2B). As shown in Fig. 2D, carvacrol (5 and 10 $\mathrm{mg} / \mathrm{kg}$ ) significantly decreased the Bax:Bcl-2 ratio in the kidney tissue in the acetaminophen + carvacrol 5 $\mathrm{mg} / \mathrm{kg}$ and acetaminophen + carvacrol $10 \mathrm{mg} / \mathrm{kg}$ groups compared to acetaminophen administered group $(P<0.05$, Fig. 2D).

\section{The effect of carvacrol on kidneys tissue damage}

After histopathologic analysis, no significant pathological changes were found in the control group (Fig. 3). The hematoxylin and eosin staining results also showed that acetaminophen $(500 \mathrm{mg} / \mathrm{kg})$ administration can increase the glomerular or tubulointerstitial damage in the kidneys tissue, so that severe kidney damage was seen in acetaminophen administered rats when compared with the control (Fig. 3A). Also, KTDS was significantly higher in the acetaminophen administrated group versus control $(p<0.001$, Fig. 3B). Carvacrol $(10 \mathrm{mg} / \mathrm{kg})$ could decrease the damage caused by acetaminophen 
administration (Fig. 3). So that, carvacrol could significantly decrease the KTDS in the acetaminophen + carvacrol $10 \mathrm{mg} / \mathrm{kg}$ group versus acetaminophen administered group $(\mathrm{P}<0.05$, Fig. 3B).

\section{Discussion}

Our result showed that a single overdose administration of acetaminophen (500 mg/ $\mathrm{kg}$ ) can decrease the RBF in rats. However, acetaminophen overdose does not alter MAP, RPP, and RVR indicators significantly (Table 1). In this regard, it has already been reported that acetaminophen administration in a dose dependent manner induced RBF and GFR impairment in rats $[25,26]$. So, this condition can affect kidney function and decrease the renal ability to concentrate urine [25]. Our study also showed that carvacrol (10 $\mathrm{mg} / \mathrm{kg}$ ) significantly can decrease the RVR and also increases RBF in the acetaminophen administered rats which were treated by carvacrol (Table 1). In confirmation of this finding, it is specified that carvacrol blocks the $\mathrm{Ca} 2+$ influx through the membrane into the cells [27] and improves blood circulation via the reduction of vascular resistance [11]. In this regard, it has also been revealed that the vasorelaxant effect of carvacrol is mediated via endothelium TRPV3 channels [11]. Our result additionally showed that serum creatinine and BUN increased in acetaminophen $(500 \mathrm{mg} / \mathrm{kg})$ administered animal. Besides, carvacrol can significantly decrease the serum creatinine and BUN in acetaminophen administered rats that were treated by carvacrol (Table 1). Acetaminophen Overdose also elevates the urea and creatinine plasma levels, a condition that is considered as drug-induced nephrotoxicity [28]. According to our study it has been determined that carvacrol administration can decrease serum creatinine and BUN in a renal ischemia-reperfusion model and subsequently reverses the pathological changes in the kidney [29]. The results of the present study also showed that a single overdose of acetaminophen $(500 \mathrm{mg} / \mathrm{kg})$ can increase the kidney tissue MDA concentration. Moreover, treatment of the rats with carvacrol $(10 \mathrm{mg} / \mathrm{kg}$ can decrease the kidney tissue MDA level in the acetaminophen overdose administered rats (Fig. 1A). Acetaminophen overdose increases the kidney and liver tissue MDA concentration as the second messenger of free radicals via depleting the antioxidant enzymes [30]. However, Bozkurt et al., (2014) revealed that carvacrol decreases serum and kidney tissue MDA levels and inhibits oxidative stress via its antioxidant effect in rats subjected to methotrexate-induced renal damage [31]. Our study also showed that taking high doses of acetaminophen can decrease the GPx and SOD activity levels in the kidneys tissue of the rats (Fig. 1B and 1C). However, treatment of the rats with carvacrol increases the GPx (carvacrol at the dose of $10 \mathrm{mg} / \mathrm{kg}$ ) and SOD (carvacrol at the dose of 5 and $10 \mathrm{mg} / \mathrm{kg}$ ) activity levels in the kidneys tissue (Fig. 1B and 1C). In this regard, Roy et al., (2015) showed that acetaminophen overdose reduces antioxidant enzymes such as SOD and GPx, thereby debilitating the antioxidant system and inducing renal oxidative injury [1]. On the other hand, Suganthi and his colleague (2013) have shown that carvacrol exerts a potent antioxidant effect via its hydroxyl group and scavenges the free radicals both in-vivo and in-vitro model [32]. Moreover, it has been reported that carvacrol ameliorates oxidative stress-induced renal injury in the ischemia-reperfusion model [29]. Our results also showed that acetaminophen at the high dose administration can increase the cleaved caspase-3, Bax proteins expression rate, and also it decreases the Bcl-2 protein expression level in the kidney tissue (Fig. 2). Based on the results of our study, carvacrol $(10 \mathrm{mg} / \mathrm{kg})$ can prevent acetaminophen-induced cleaved caspase-3 
and Bax proteins expression rate in the kidneys tissue (Fig. 2A and 2B). Furthermore, carvacrol (5 and 10 $\mathrm{mg} / \mathrm{kg}$ ) could decrease the Bax:Bcl-2 ratio in the kidneys tissue in the acetaminophen overdose administered rats (Fig. 2D). In line with the present study, it has been determined that acetaminophen can induce renal tubular cell apoptosis (even at a therapeutic dose) via activation of the caspase-9 and caspase-3 and cytochrome c cascade mechanism [2]. However, Potočnjak and Domitrović (2016) have shown that Carvacrol can ameliorate cisplatin-induced renal injury via its anti-apoptotic (upregulation of $\mathrm{Bcl}-2$ protein and downregulation of Bax proteins expression rate) effects [33]. Our results also showed that taking high doses of acetaminophen can increase kidney tissue damage (Fig. 3). But, carvacrol (10 $\mathrm{mg} / \mathrm{kg}$ ) can decrease the damage caused by overdose acetaminophen administration (Fig. 3). Consistent with our study, it has been shown that acetaminophen overdose causes kidney injury via tubular necrosis and glomerular damages [1] which is consistent with our result of the renal histopathological change. In this regard, Gunes et al. (2017) reported that carvacrol improves kidney histology and ameliorates cyclophosphamide-induced renal damage [15]. Moreover, El-Sayed et al. (2015) showed that carvacrol attenuates cisplatin-induced renal injury via glomerular and tubular necrosis reduction [34].

\section{Conclusion}

Based on our findings, it seems that carvacrol $(10 \mathrm{mg} / \mathrm{kg})$ has anti-apoptotic properties in the kidney tissue against overdose acetaminophen administration in rats. Moreover, carvacrol can improve kidney function in acetaminophen overdose administered animals. Also, carvacrol can reduce lipid peroxidation level probably through scavenging free radicals in kidney tissue of acetaminophen overdose administered animal. Furthermore, carvacrol can reduce kidney damage and improves the renal dysfunction in this model. Totally, carvacrol may be used as a kidney damage reducer after acetaminophen overdose administration in the future. However, further study is needed.

\section{Declarations}

\section{Acknowledgement}

This study was supported by Rafsanjan University of Medical Sciences (Grant \# 97301).

\section{Compliance with ethical standards}

The authors declare that there are no conflicts of interest.

\section{Author contributions}

Conceived and designed the experiments: JH. Performed the experiments: AK, JH, MR, and AN. Analyzed the data: JH and MR. Contributed reagents/materials/analysis tools: $\mathrm{EH}$, and JH. Wrote the paper: AN, JH and AK.

\section{Consent to Participate}


All authors were voluntarily agreed to participate in this study.

\section{Consent to Publish}

All authors were voluntarily agreed to publish the results of this study

\section{References}

1. Roy S, Pradhan S, Das K, Mandal A, Mandal S, Patra A, Samanta A, Sinha B, Nandi DK (2015) Acetaminophen induced kidney failure in rats: A dose response study. Journal of Biological Sciences 15(4):187. doi:10.3923/jbs.2015.187.193

2. Lorz C, Justo P, Sanz A, Subirá D, Egido J, Ortiz A (2004) Paracetamol-induced renal tubular injury: a role for ER stress. J Am Soc Nephrol 15 (2):380-389. 10.1097/01.asn. doi:0000111289.91206.b0

3. Aycan iö, Tokgöz O, Tüfek A, Alabalık U, Evliyaoğlu O, Turgut H, Çelik F, Güzel A (2015) The use of thymoquinone in nephrotoxicity related to acetaminophen. Int J Surg 13:33-37.

doi:10.1016/j.ijsu.2014.11.020

4. Mazer M, Perrone J (2008) Acetaminophen-induced nephrotoxicity: pathophysiology, clinical manifestations, and management. J Med Toxicol 4(1):2-6. doi:10.1007/BF03160941

5. El-Shafey MM, Abd-Allah GM, Mohamadin AM, Harisa GI, Mariee AD (2015) Quercetin protects against acetaminophen-induced hepatorenal toxicity by reducing reactive oxygen and nitrogen species. Pathophysiology 22(1):49-55. doi:10.1016/j.pathophys

6. Kaeidi A, Sahamsizadeh A, Allahtavakoli M, Fatemi I, Rahmani M, Hakimizadeh E, Hassanshahi J (2020) The effect of oleuropein on unilateral ureteral obstruction induced-kidney injury in rats: the role of oxidative stress, inflammation and apoptosis. Molecular biology reports 47(2):1371-1379. doi:10.1007/s11033-019-05237-0

7. Kaeidi A, Taghipour Z, Allahtavakoli M, Fatemi I, Hakimizadeh E, Hassanshahi J (2020) Ameliorating effect of troxerutin in unilateral ureteral obstruction induced renal oxidative stress, inflammation, and apoptosis in male rats. Naunyn Schmiedebergs Arch Pharmacol 393(5):879-888.

doi:10.1007/s00210-019-01801-4

8. Hassanshahi J, Mirzahosseini-Pourranjbar A, Hajializadeh Z, Kaeidi A (2020) Anticancer and cytotoxic effects of troxerutin on HeLa cell line: an in-vitro model of cervical cancer. Mol Biol Rep 47(8):6135-6142. doi:10.1007/s11033-020-05694-y

9. Cai Y, Luo Q, Sun M, Corke H (2004) Antioxidant activity and phenolic compounds of 112 traditional Chinese medicinal plants associated with anticancer. Life sciences 74(17):2157-2184. doi:10.1016/j.lfs.2003.09.047

10. Fukumoto L, Mazza G (2000) Assessing antioxidant and prooxidant activities of phenolic compounds. J Agric Food Chem 48(8):3597-3604. doi:10.1021/jf000220w

11. Suntres ZE, Coccimiglio J, Alipour M (2015) The bioactivity and toxicological actions of carvacrol. Critical reviews in food science nutrition 55(3):304-318. doi:10.1080/10408398.2011.653458 
12. Kohen R, Nyska A (2002) Invited review: Oxidation of biological systems: oxidative stress phenomena, antioxidants, redox reactions, and methods for their quantification. Toxicol Pathol 30(6):620-650. doi:10.1080/01926230290166724

13. Melo FHC, Moura BA, de Sousa DP, de Vasconcelos SMM, Macedo DS, Fonteles MMdF, Viana GSdB, de Sousa FCF (2011) Antidepressant-like effect of carvacrol (5-Isopropyl-2-methylphenol) in mice: involvement of dopaminergic system. Fundam Clin Pharmacol 25(3):362-367. doi:10.1111/j.14728206.2010.00850.x

14. Uyanoglu M, Canbek M, Ceyhan E, Senturk H, Bayramoglu G, Gunduz O, Ozen A, Turgak O (2011) Preventing organ injury with carvacrol after renal ischemia/reperfusion. Journal of Medicinal Plants Research 5(1):72-80

15. Gunes S, Ayhanci A, Sahinturk V, Altay DU, Uyar R (2017) Carvacrol attenuates cyclophosphamideinduced oxidative stress in rat kidney. Can J Physiol Pharmacol 95(7):844-849. doi:10.1139/cjpp2016-0450

16. Hassanshahi J, Nematbakhsh M (2018) The role of Mas receptor on renal hemodynamic responses to angiotensin 1-7 in both irreversible and reversible unilateral ureteral obstruction rats. Advanced biomedical research 7. doi:10.4103/abr

17. Samimiat A, Khosravi MS, Hassanshahi J, Nematbakhsh M (2018) The effect of AT2 and Mas receptors antagonists on renal hemodynamic and excretory disorders induced by ischemia/reperfusion in male and female rats. Physiology Pharmacology 22(2):133-140

18. Hantz H, Adesuyi A, Adebayo O (2001) Differential effects of U46619 on renal regional hemodynamics in the rat: involvement of endothelin. J Pharmacol Exp Ther 299(1):372-376

19. Hassanshahi J, Maleki M, Nematbakhsh M (2018) Renal blood flow and vascular resistance responses to angiotensin II in irreversible and reversible unilateral ureteral obstruction rats; the role of angiotensin II type 1 and 2 receptors. Journal of nephropathology 7 (2)

20. Richard MJ, Portal B, Meo J, Coudray C, Hadjian A, Favier A (1992) Malondialdehyde kit evaluated for determining plasma and lipoprotein fractions that react with thiobarbituric acid. Clinical chemistry 38(5):704-709

21. Ōyanagui Y (1984) Reevaluation of assay methods and establishment of kit for superoxide dismutase activity. Anal Biochem 142(2):290-296. doi:10.1016/0003-2697(84)90467-6

22. Flohé L, Günzler WA (1984) [12] Assays of glutathione peroxidase. Methods Enzymol 105:114-120

23. Wang B, Liu D, Zhu Q-h, Li M, Chen H, Guo Y, Fan L-p, Yue L-s, Li L-y, Zhao M (2016) Rutin ameliorates kidney interstitial fibrosis in rats with obstructive nephropathy. Int Immunopharmacol 35:77-84. doi:10.1016/j.intimp.2016.03.029

24. Sancak EB, Tan YZ, Turkon H, Silan C (2017) Attenuation of partial unilateral ureteral obstructioninduced renal damage with hyperbaric oxygen therapy in a rat model. International braz $\mathrm{j}$ urol 43(5):946-956. doi:10.1590/S1677-5538.IBJU.2016.0565

25. Pakravan N, Bateman DN, Goddard J (2007) Effect of acute paracetamol overdose on changes in serum and urine electrolytes. Br J Clin Pharmacol 64(6):824-832. doi:10.1111/j.1365- 
26. Trumper L, Girardi G, Elías MM (1992) Acetaminophen nephrotoxicity in male Wistar rats. Arch Toxicol 66(2):107-111

27. Peixoto-Neves D, Silva-Alves K, Gomes M, Lima F, Lahlou S, Magalhães P, Ceccatto V, Coelho-deSouza A, Leal-Cardoso J (2010) Vasorelaxant effects of the monoterpenic phenol isomers, carvacrol and thymol, on rat isolated aorta. Fundam Clin Pharmacol 24(3):341-350. doi:10.1111/j.14728206.2009.00768.x

28. Mandal A, Patra A, Mandal S, Roy S, Mahapatra SD, Mahapatra TD, Paul T, Das K, Mondal KC, Nandi DK (2015) Therapeutic potential of different commercially available synbiotic on acetaminopheninduced uremic rats. Clin Exp Nephrol 19(2):168-177. doi:10.1007/s10157-014-0971-4

29. Gheitasi I, Azizi A, Omidifar N, Doustimotlagh AH (2020) Renoprotective Effects of Origanum majorana Methanolic $L$ and Carvacrol on Ischemia/Reperfusion-Induced Kidney Injury in Male Rats. Evidence-Based Complementary and Alternative Medicine 2020

30. Yoon E, Babar A, Choudhary M, Kutner M, Pyrsopoulos N (2016) Acetaminophen-induced hepatotoxicity: a comprehensive update. Journal of clinical translational hepatology 4(2):131. doi:10.14218/JCTH.2015.00052

31. Bozkurt M, Em S, Oktayoglu P, Turkcu G, Yuksel H, Sarıyıldız MA, Caglayan M, Batmaz İ, Nas K, Bozkurt Y (2014) Carvacrol prevents methotrexate-induced renal oxidative injury and renal damage in rats. Clinical and Investigative Medicine:E19-E25. doi: 10.25011/cim.v37i1.20865

32. Suganthi RU, Manpal S (2013) Biological and pharmacological of actions carvacrol and its effects on poultry: An updated review. World J Pharm Pharm Sci 2 (2013):3581-3595

33. Potočnjak I, Domitrović R (2016) Carvacrol attenuates acute kidney injury induced by cisplatin through suppression of ERK and PI3K/Akt activation. Food Chem Toxicol 98:251-261. doi:10.1016/j.fct.2016.11.004

34. EM ES, AR AA, AM M, AA EA (2015) Thymol and carvacrol prevent cisplatin-induced nephrotoxicity by abrogation of oxidative stress, inflammation, and apoptosis in rats. J Biochem Mol Toxicol 29(4):165-172. doi:10.1002/jbt.21681

\section{Tables}

Table 1. The body weight, renal hemodynamic parameters values, BUN and creatinine levels according to the groups $(n=8)$ at the end of experiment. All statistical data was analyzed by one-way ANOVA follow-

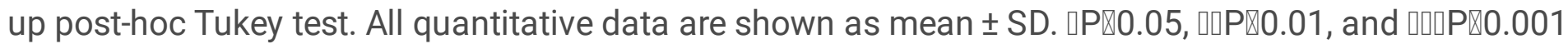
compared to control. \#P $₫ 0.05$, and \#\#\#P®0.001 versus acetaminophen group. MAP: Mean arterial pressure, RBF: Renal blood flow, RPP: Renal perfusion pressure, RVR: Renal vascular resistance, Cr: Creatinine, BUN: Blood urea nitrogen. 


\begin{tabular}{|c|c|c|c|c|c|c|c|}
\hline \multirow[t]{2}{*}{ Groups } & \multirow[t]{2}{*}{ Body weight (g) } & \multicolumn{4}{|c|}{ Hemodynamic parameters } & \multicolumn{2}{|c|}{ Function parameters } \\
\hline & & $\begin{array}{c}\text { MAP } \\
(\mathrm{mmHg})\end{array}$ & $\begin{array}{c}\text { RPP } \\
(\mathrm{mmHg})\end{array}$ & $\begin{array}{l}\text { PBF } \\
\text { (PU) }\end{array}$ & $\begin{array}{c}\text { RVR } \\
(\mathrm{mmHg} / \mathrm{PU})\end{array}$ & $\begin{array}{l}\text { Serum Cr } \\
\text { (mg/dl) }\end{array}$ & $\begin{array}{c}\text { BUN } \\
\text { (mg/dl) }\end{array}$ \\
\hline Control & $218 \pm 3.25$ & $106 \pm 3.80$ & $94 \pm 6.18$ & $155 \pm 11.62$ & $0.63 \pm 0.07$ & $0.38 \pm 0.06$ & $36 \pm 8.21$ \\
\hline Carvacrol $10 \mathrm{mg} / \mathrm{kg}$ & $214 \pm 4.45$ & $100 \pm 6.39$ & $95 \pm 7.13$ & $151 \pm 12.93$ & $0.65 \pm 0.06$ & $0.40 \pm 0.08$ & $38 \pm 7.45$ \\
\hline Acetaminophen & $211 \pm 4.92$ & $103 \pm 4.65$ & $96 \pm 5.54$ & $123 \pm 10.24^{*}$ & $0.78 \pm 0.10$ & $1.04 \pm 0.13^{* * *}$ & $62 \pm 9.56^{* *}$ \\
\hline Acetaminophen + Carvacrol $5 \mathrm{mg} / \mathrm{kg}$ & $209 \pm 6.13$ & $104 \pm 3.17$ & $94 \pm 3.36$ & $139 \pm 15.11$ & $0.68 \pm 0.10$ & $0.81 \pm 0.12$ & $46 \pm 9.32$ \\
\hline Acetaminophen + Carvacrol $10 \mathrm{mg} / \mathrm{kg}$ & $215 \pm 2.98$ & $105 \pm 6.18$ & $89 \pm 2.75$ & $150 \pm 12.67 \mathrm{H}$ & $0.59 \pm 0.06 \#$ & $0.57 \pm 0.11 \mathrm{HAN}$ & $42 \pm 8.18 \#$ \\
\hline
\end{tabular}

Figures

A

B
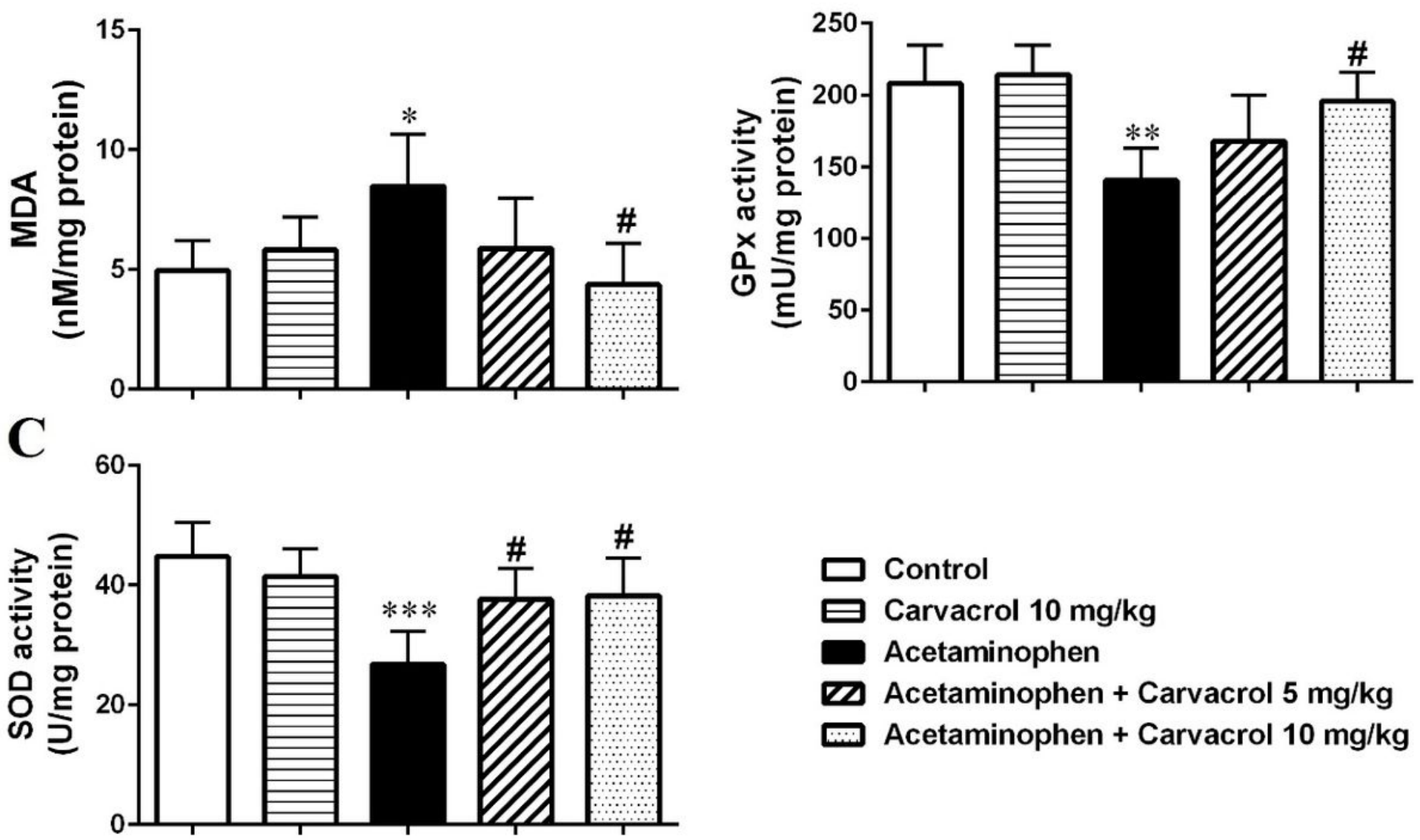

Figure 1

The serum MDA concentration, GPx and SOD activity levels as three oxidative stress parameters according to the groups $(n=8)$ at the end of experiment. All statistical data was analyzed by one-way ANOVA follow-up post-hoc Tukey test. All quantitative data are shown as mean \pm SD. $\square P \llbracket 0.05, \square[P \llbracket 0.01$, 
and $\triangle \mathrm{P} \otimes 0.001$ versus control group. \#Pख0.05 versus acetaminophen group. MDA: malondialdehyde, SOD: superoxide dismutase, GPx: glutathione peroxidase.
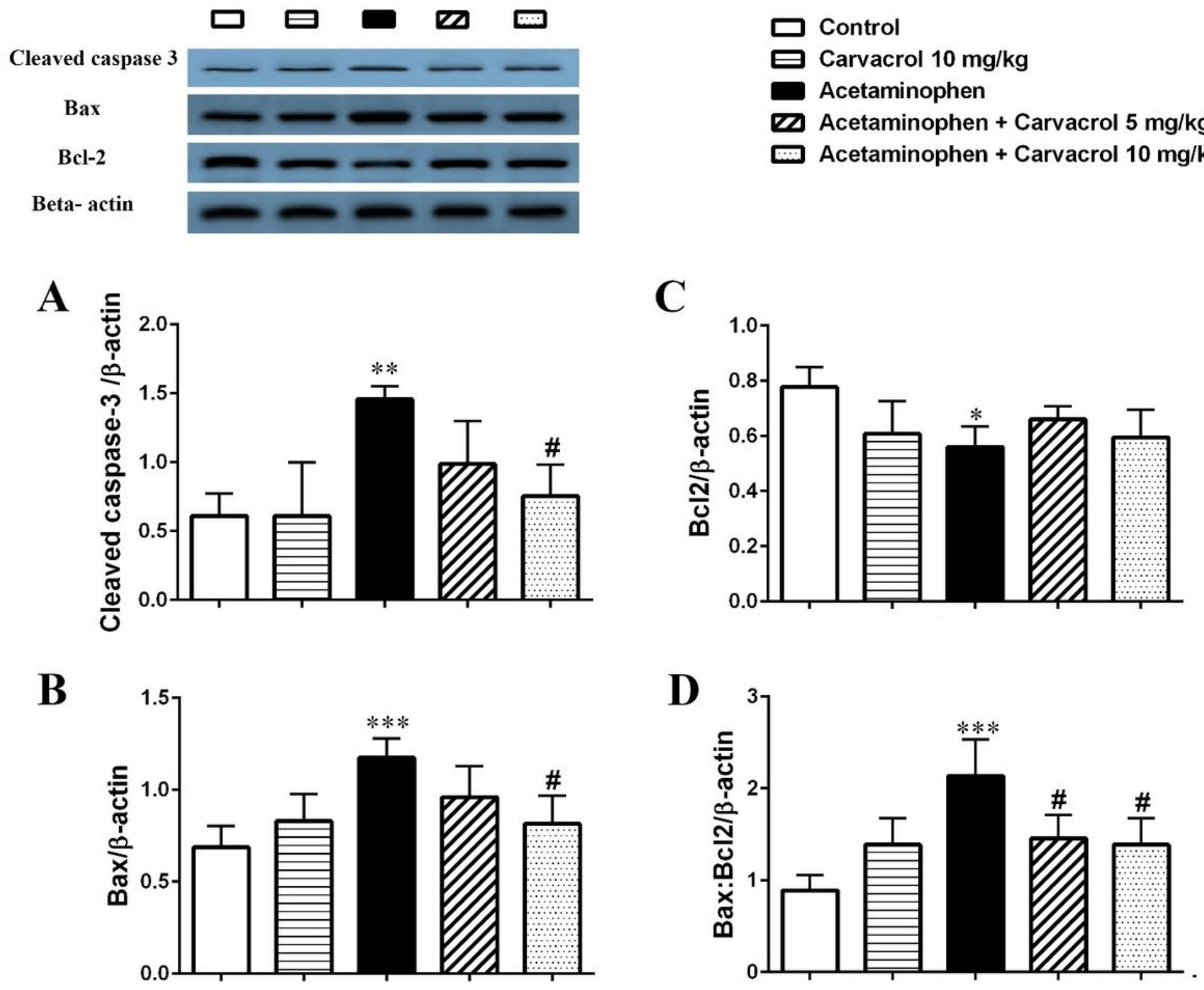

Figure 2

the western blotting analysis of cleaved caspase-3 (A), Bax (B), Bcl-2 (C), and Bax/Bcl-2 ratio (D) proteins as critical parameters of the apoptotic pathway according to the groups $(n=8)$ at the end of experiment. All statistical data was analyzed by one-way ANOVA follow-up post-hoc Tukey test. All quantitative data

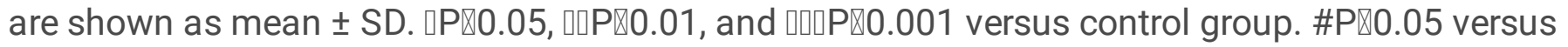
acetaminophen group. 


\section{A}

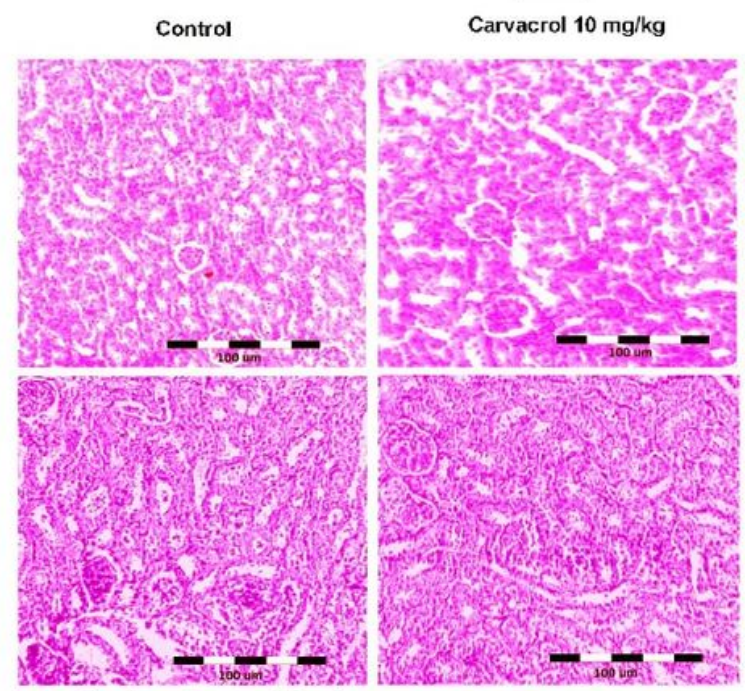

Acetaminophen + Carvacrol $5 \mathrm{mg} / \mathrm{kg}$ Acetaminophen + Carvacrol $10 \mathrm{mg} / \mathrm{kg}$

\section{B}

$\square$ Control

口 Carvacrol $10 \mathrm{mg} / \mathrm{kg}$

- Acetaminophen

ש Acetaminophen + Carvacrol $5 \mathrm{mg} / \mathrm{kg}$

西 Acetaminophen + Carvacrol $10 \mathrm{mg} / \mathrm{kg}$

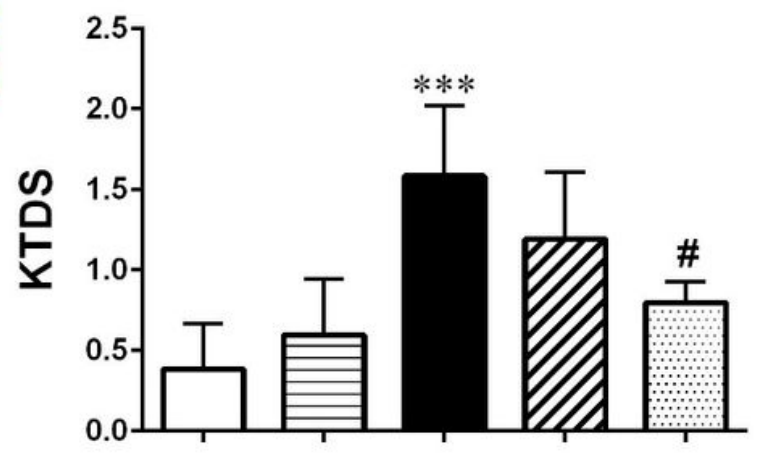

Figure 3

The hematoxylin and eosin stained sections (magnification 100x) (A) and Kidney tissue damage score (B) in all groups $(n=8)$ at the end of experiment. All statistical data was analyzed by Kruskal-Wallis test. Data are shown as mean \pm SD. $00 \mathrm{P} \llbracket 0.001$ versus control group. \#Pख0.05 versus acetaminophen group. KTDS: Kidney tissue damage score. 